\title{
The circlet transform: A robust tool for detecting features with circular shapes
}

\author{
H. Chauris ${ }^{a, b,{ }^{*}}$, I. Karoui ${ }^{a, c}$, P. Garreau ${ }^{c}$, H. Wackernagel ${ }^{a}$, P. Craneguy ${ }^{d}$ and L. Bertino ${ }^{e}$ \\ a Centre de Géosciences, Mines ParisTech, 35 rue Saint-Honoré, 77300 Fontainebleau, France \\ ${ }^{\mathrm{b}}$ UMR-Sisyphe 7619, UPMC, Paris, France \\ c Ifremer, Plouzané, France \\ d Actimar, Brest, France \\ e Nersc, Bergen, Norway \\ *Corresponding author : H. Chauris, email address : herve.chauris@mines-paristech.fr
}

\begin{abstract}
:
We present a novel method for detecting circles on digital images. This transform is called the circlet transform and can be seen as an extension of classical 1D wavelets to 2D; each basic element is a circle convolved by a 1D oscillating function. In comparison with other circle-detector methods, mainly the Hough transform, the circlet transform takes into account the finite frequency aspect of the data; a circular shape is not restricted to a circle but has a certain width. The transform operates directly on image gradient and does not need further binary segmentation. The implementation is efficient as it consists of a few fast Fourier transforms. The circlet transform is coupled with a soft-thresholding process and applied to a series of real images from different fields: ophthalmology, astronomy and oceanography. The results show the effectiveness of the method to deal with real images with blurry edges.
\end{abstract}

Keywords: Circlet transform; Circle detection; Image processing; Multi-scale representation; Computer vision 


\section{Introduction}

The detection of circular structures is a key factor in a number of computer vision applications, especially in medical imaging and robotics. The objective is for example to derive automatic inspections or to extract features from an image or from a sequence of images (Mallat, 1996; Davies, 1997). More specific references related to circle detection can be found in Lam and Yuen (1996); Ayala-Ramirez et al. (2006). Within the deterministic approaches, the geometric hashing techniques try to match geometrical features against a database (Iivarinen et al., 1997). The Hough transform (HT) is an widely used alternative method to find parametrized shapes in digital images (Duda and Hart, 1972; Ballard, 1981). For a review, we refer to Maître (1985); Leavers (1993); Illingworth and Kittler (1988); Song and Lyu (2005). The HT is known to be robust against noise, but the classical implementation requires massive computation and memory. More importantly, it does not take into account the width of the contours. The band-limited aspect of the data is a key element that should be considered (Kiryati and Bruckstein, 1991).
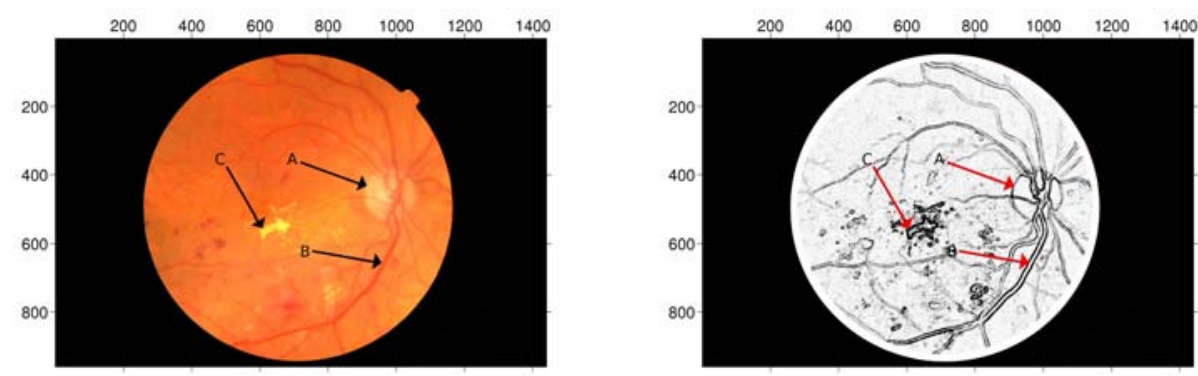

Figure 1: Retinography image (left) and the associated image gradient (right), with A: optic disk, B: retinal blood vessels, and C: exudates. The local gradient values are higher around B and C. Only the optic disk has a circular shape.

In this introduction, we select a particular example in the medical imaging domain to motivate the development of a new transform. Other applications in different fields are later introduced. In the eye fundus image (Fig. 1, left), one could distinguish between the optic disk with a circular shape (A), the retinal blood vessels (B) and exudates (C). Glaucoma neuropathy refers to damages of the optic nerves and can lead to loss of vision. In that context, the evaluation of the size of the optic disk is of first importance (Montgomery, 
1993). The particular eye (Fig. 1) shows hard exudates with a possible large risk of macular edema. The objective is to detect the optic disk that should not be mistaken for exudates nor for vessels.
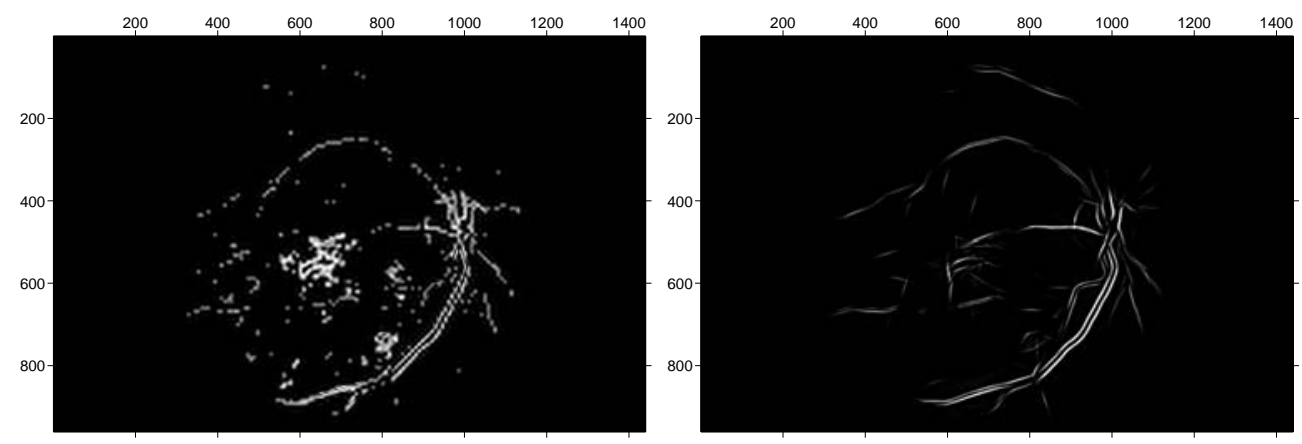

Figure 2: Reconstructed image after Meyer wavelet (left) and curvelet (right) transforms. In each case, only coefficients with values higher than $40 \%$ of the maximum value are selected. Other coefficients are set to zero.

In order to emphasize the contrast in the eye image $f(x, y)$ where $(x, y)$ denotes the spatial coordinates, the processing is usually performed on the image gradient $|\nabla f|$ (Fig. 1, right). It appears that the contrast at the exudates and along the blood vessels are larger than the contrast around the optic disk. With the multi-scale aspect, the width of the contours is taken into account in the wavelet and curvelet transforms, not in the classical HT. A wavelet analysis (Daubechies, 1992; Mallat, 1996) first reveals the positions of the exudates with their irregular shapes (Fig. 2, left). Classical 2D wavelets or oscillatory functions are indeed very useful to detect point singularities. Vessels are rather associated to a relatively smooth curve. For that, curvelets are well suited for the detection (Candes and Donoho, 2004; Candes et al., 2005) (Fig. 2, right). Curvelets can be seen as an extension of wavelets where the elements used for the decomposition are elongated along one axis and oscillating in the perpendicular direction. We refer to $\mathrm{Ma}$ and Plonka (2010) for a review on curvelet applications. Both the wavelet and curvelet analysis do not reveal the optic disk. The circlet transform proposed here has been designed to detect objects with circular shapes. It should be understood as an alternative to wavelets and curvelets, still with the multiscale aspect, but also with the possibility to follow global structures as for the HT.

The objective of the new transform for detecting circular shapes should 
address the following issues: (1) the user should be able to specify a range of radii for the selection; (2) the segmentation part should be avoided for more robustness; (3) the circular shape should be an annulus of a certain width in order to handle non perfectly circular shapes. This is also a way to take into account the band-limited aspect of the data; (4) the transform should be perfectly invertible: if all coefficients are selected, then the inverted image should be the same as the original image; (5) the transform should be fast enough to enable iterative processes such as the soft-thresholding technique for reducing the number of key elements needed to represent the circular structures in the image (Donoho, 1995).

The outline of the paper is the following. We first describe the circlet transform. We then present the algorithm used to select only a few representative circlet coefficients. We show applications in three different fields: ophthalmology, moon exploration and coastal oceanography, with mainly real images. The applications range from the detection of a single and clear circular shape to the finding of a series of circular shapes with more diffuse contours. Finally, we discuss the current limitations of the method and what could be modified, in particular to remove some spurious detections.

\section{The circlet transform}

In the proposed approach, there is no need for binary image segmentation. The method consists of decomposing any image into "circles" with different radii and a certain width, via a series of Fast Fourier Transforms (FFTs). These circles are called circlets as they can be seen as the convolution of a circle with a 1D oscillatory function, possibly a wavelet, in the same way as wavelets relate to waves. However, the strategy for computing the circlet coefficients is rather different from the classical approach used for wavelet decomposition. Usually, wavelet coefficients are obtained via a series of cascaded convolutions and down-sampling (Daubechies, 1992). As explained bellow, the circlet decomposition is formulated in the Fourier domain with the definition of specific filters, following a similar approach as the one proposed by Candes et al. (2005) for curvelets.

\subsection{General framework}

The circlet elements are characterized by a central position $\left(x_{0}, y_{0}\right)$, a radius $r_{0}$ and a central frequency content $f_{0}$ (Fig. 3). This finite frequency $f_{0}$ provides a certain width to the circlet in the spatial domain. This is 
the main difference with the Hough transform, beyond the implementation aspects. All circlet elements $c_{\mu}(x, y)$ can be deduced from a reference circlet $c_{\text {ref }}(x, y)$, either by a shift or by modifying the radius or the central frequency content of the circlet (Fig. 4, Eq. 1). The parameters $\mu=\left(x_{0}, y_{0}, r_{0}, f_{0}\right)$ fully characterize each circlet. Formally, the circlet function can be written as

$$
c_{\mu}(x, y)=\Omega\left[2 \pi f_{0}\left(r-r_{0}\right)\right],
$$

where $r=\sqrt{\left(x-x_{0}\right)^{2}+\left(y-y_{0}\right)^{2}}$. $\Omega$ is typically an oscillatory function, possibly a wavelet function, designed to detect discontinuities (Daubechies, 1992). From a practical point of view, $c_{\mu}$ will be explicitly defined in the $2 \mathrm{D}$ Fourier domain.
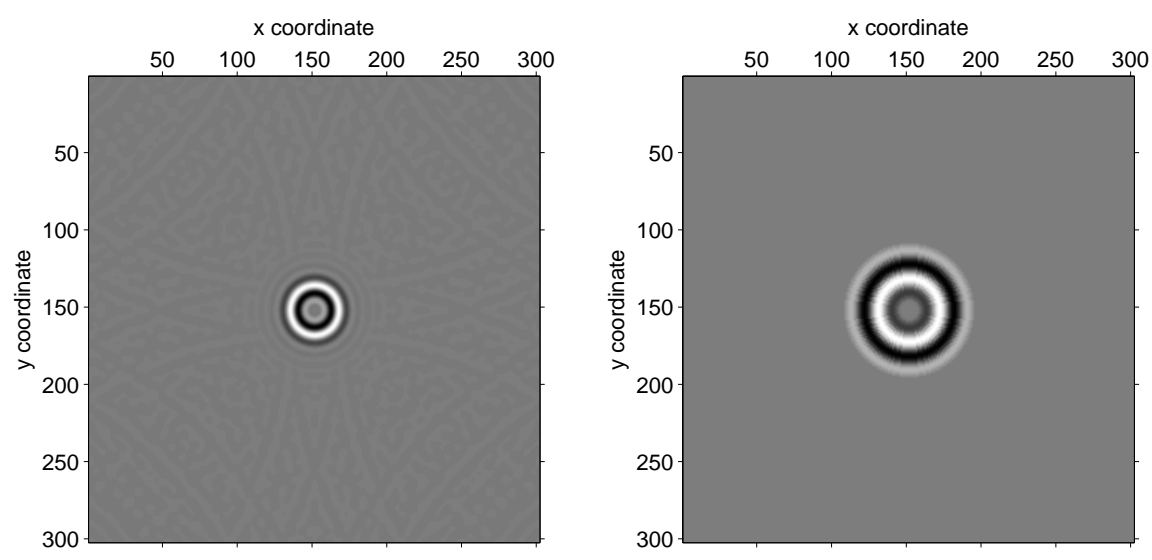

Figure 3: Representation of a single circlet (left) and its 2D Fourier transform (right). By construction, it is well-localized in the Fourier domain.

\subsection{The circlet decomposition}

The forward and inverse transform is similar in essence to the curvelet transform (Candes and Donoho, 2004; Candes et al., 2005). The objective is to decompose any $2 \mathrm{D}$ image $f(x, y)$ into a sum of basic functions $c_{\mu}$ :

$$
f(x, y)=\sum_{\mu} A_{\mu} \cdot c_{\mu}(x, y)
$$

For curvelets, the basic elements have elongated shapes, similar to the representation of local plane waves (Candes et al., 2005). For circlets, the 

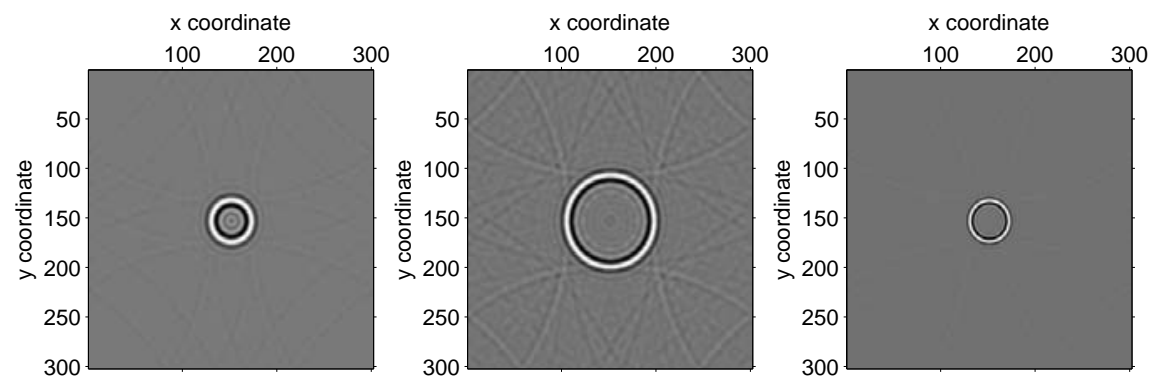

Figure 4: Representation of the reference circlet (left) and other circlets corresponding to a different radius (middle) and to a different frequency content (right). The artifacts visible on the image in the middle are due to an abrupt truncation in the shape of the $F_{k}$ filters (Fig. 5).

basic functions are circular. The circlet construction uses the properties of a tight frame, so that the associated amplitudes $A_{\mu}$ are obtained by a scalar product

$$
A_{\mu}=<f, c_{\mu}>=\iint \mathrm{d} x \mathrm{~d} y f(x, y) \cdot c_{\mu}(x, y) .
$$

From a practical point of view, the circlet coefficients are defined in the Fourier domain, using Parseval's theorem

$$
A_{\mu}=<\hat{f}, \hat{c}_{\mu}>=\iint \mathrm{d} \omega_{1} \mathrm{~d} \omega_{2} \hat{f}\left(\omega_{1}, \omega_{2}\right) \cdot \hat{c}_{\mu}^{*}\left(\omega_{1}, \omega_{2}\right),
$$

where $\hat{f}$ denotes the 2D Fourier transform of $f$ and $f^{*}$ the conjugate of $f$. With this formulation, the circlet transform is constructed in the 2D Fourier domain. The key step consists of defining $\hat{c}_{\mu}^{*}\left(\omega_{1}, \omega_{2}\right)$, the Fourier transform of $c_{\mu}$. This is obtained by developing appropriate filters to ensure that the basic functions $c_{\mu}(x, y)$ have circular shapes.

\subsection{Definition of filters}

The filter construction is obtained in a two-step process: first we derive 1D filters $F_{k}$ and then $2 \mathrm{D}$ filters $G_{k}$. Both filters $F_{k}$ and $G_{k}$ are defined in the frequency domain and form a partition of it: for all $\omega$ and $\left(\omega_{1}, \omega_{2}\right)$, we have 


$$
\begin{aligned}
\sum_{k}\left|F_{k}(\omega)\right|^{2} & =1 \\
\sum_{k}\left|G_{k}\left(\omega_{1}, \omega_{2}\right)\right|^{2} & =1
\end{aligned}
$$

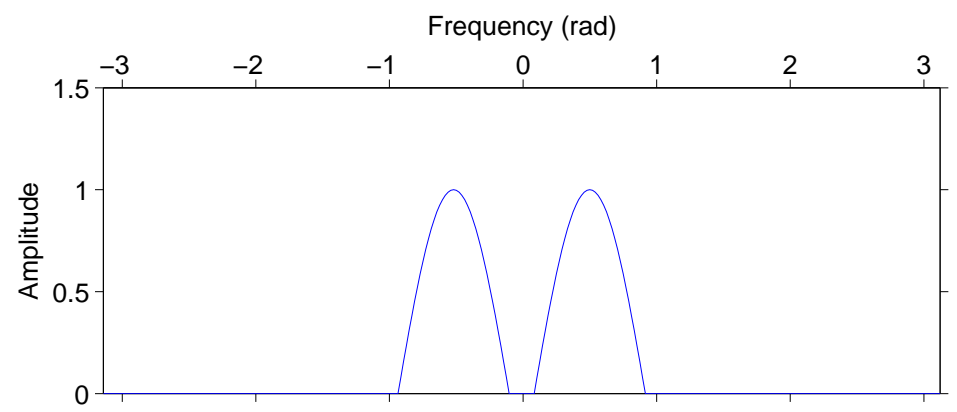

Figure 5: Representation of a single filter $F_{k}$ for $k=2$ determining the frequency content of the circlet.

This condition is important to ensure a perfect reconstruction scheme (Candes et al., 2005). First define $\omega_{k}=\pi(k-1) /(N-1)$ where $N$ is the number of filters. For $\left|\omega \pm \omega_{k}\right| \leq \pi /(N-1), F_{k}(\omega)=\cos \left(\omega \pm \omega_{k}\right)$, otherwise $F_{k}=0$. Note that the $F_{k}$ filters are symmetric (Fig. 5). One can easily check that the $F_{k}$ filters form a partition of the $1 \mathrm{D}$ frequency domain. The $G_{k}$ filters are defined from the $F_{k}$ filters by introducing a phase delay in order to create a circular shape in the space domain,

$$
G_{k}\left(\omega_{1}, \omega_{2}\right)=e^{i|\omega| r_{0}} \cdot F_{k}(|\omega|),
$$

where $\omega=\left(\omega_{1}, \omega_{2}\right)$ and $|\omega|=\sqrt{\omega_{1}^{2}+\omega_{2}^{2}}$. Once the filters $G_{k}$ are defined, equation 8 provides an explicit formulation for the Fourier transform of a circlet

$$
\hat{c}_{\mu}(\omega)=e^{i<\omega, x_{c}>} \cdot G_{k}(\omega),
$$

where $x_{c}=\left(x_{0}, y_{0}\right)$ is the central position and where $r_{0}$ determines the radius of the circlet. By definition, $<,>$ denotes the scalar product. We thus have $\mu=\left(x_{c}, r_{0}, k\right)$. The index $k$ controls the frequency content of the circlet 
(Fig. 4). With equations 5 and 7, it is also easy to see that for any given $r_{0}$ values, the $G_{k}$ filters also form a partition of the 2D frequency domain. By using polar coordinates, we show in appendix A that the 2D inverse Fourier Transform of $G_{k}$ is circular, meaning that the basis functions $c_{\mu}(x, y)$ have circular shapes.

\subsection{Practical implementation}

The forward circlet transform consists of (1) a 2D Fourier transform of the original image $f(x, y)$ to obtain $\hat{f}\left(\omega_{1}, \omega_{2}\right)$; (2) for all filters and for all selected

$r_{0}$ values, a multiplication of $\hat{f}$ by $G_{k}$; (3) the inverse Fourier transform of the product that provides all the circlet coefficients related to scale $k$ and radius $r_{0}$.

The inverse transform follows the same rule. First apply a 2D Fourier transform for all scales and selected radii, multiply by the conjugate of $G_{k}$ and sum all results. The final image is obtained by applying a 2D inverse Fourier transform. Because of the condition on $G_{k}$ (Eq. 6), we have a perfect reconstruction scheme if all circlet coefficients are preserved. For more details on the forward and inverse transform, we refer to the curvelet transform (Candes et al., 2005). The main difference with the circlet construction is the choice of the filters $G_{k}$. From a practical point of view, we rather select a single scale (i.e. single $F_{k}$ filter) and a series of radii, with expected values from $r_{\min }>0$ to $r_{\max }$ to potentially emphasize circular forms with some specific spatial sizes.

\section{Applications}

We first indicate how to choose the key circlet coefficients and then present applications to different fields.

\subsection{Selection of representative circlets}

Fig. 6 illustrates a very simple example. The input image consists of rectangular and circular shapes. The coefficients with the highest absolute amplitudes are associated with circular shapes. The selection of these coefficients leads to an image freed from the rectangles. In the circlet domain, the coordinates of the coefficients directly indicate the centers of circles and their associated radii. 

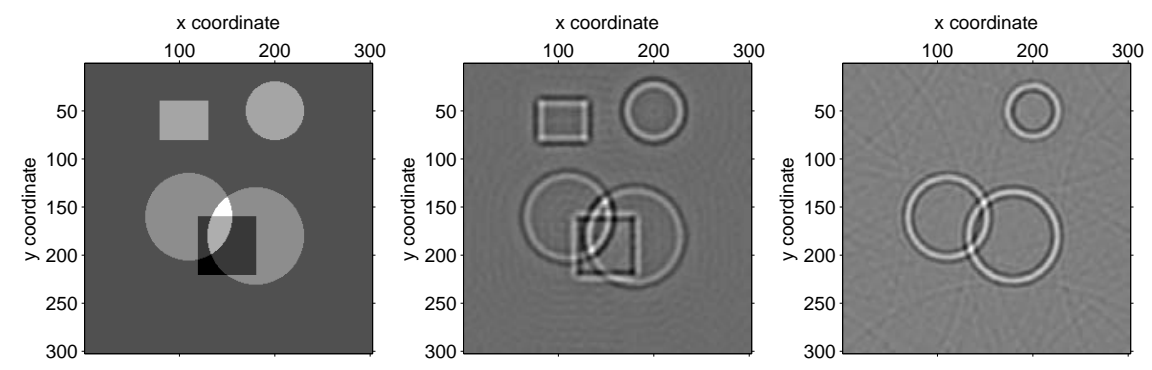

Figure 6: Simple input image (left), image reconstructed after the selection of all coefficients corresponding to a single $k=2$ value, and radius values in $[10,55]$ pixels (middle), and image after selection of $0.01 \%$ of the coefficients with the highest amplitudes.

For more complicated cases where the contours are diffuse, we apply the following strategy. As a pre-processing step, perform a spatial gradient (discrete Laplacian operator) to the original image in order to emphasize the discontinuities in the data. In the case of satellite images, they classically suffer from missing information due to the presence of clouds. We then interpolate the data by a geostatistical filtering method (kriging) that provides results spatially consistent with the original data (Wackernagel, 2003).

For the detection of a single circular structure, we simply select the highest circlet coefficient. For finding more circles, we used the soft-thresholding approach in an iterative process (Donoho, 1995). First, we select the highest coefficient. The circlet transform is redundant, meaning that the number of coefficients is larger than the size of the input data. The next coefficients with high values have approximately the same radius and central positions. For that reason, we set to zero all coefficients associated to the same radius and spatially close to the selected coefficient. By close, we mean a distance lower than half of the radius. It does not prevent from selecting a coefficient with a different radius around the same central position. We then recompose/decompose the image to obtain new coefficients. The operation is repeated until the number of key coefficients specified by the user is reached. The combination of reconstruction and decomposition is not necessarily needed. It is used here because the transform is redundant: different combinations of coefficients may represent the same image. This iterative approach is feasible with the use of FFTs.

For the different applications, we specify the radius ranges in the legend 
of the figures.

\subsection{Ophthalmology}

The motivations have been presented in the introduction part. In the first example (Fig. 7), the optic disk is clearly defined and the algorithm easily detects it. In Fig. 7 (bottom), the bright zone within the optic disk is not selected as the minimum radius is set to $30 \mathrm{px}$.
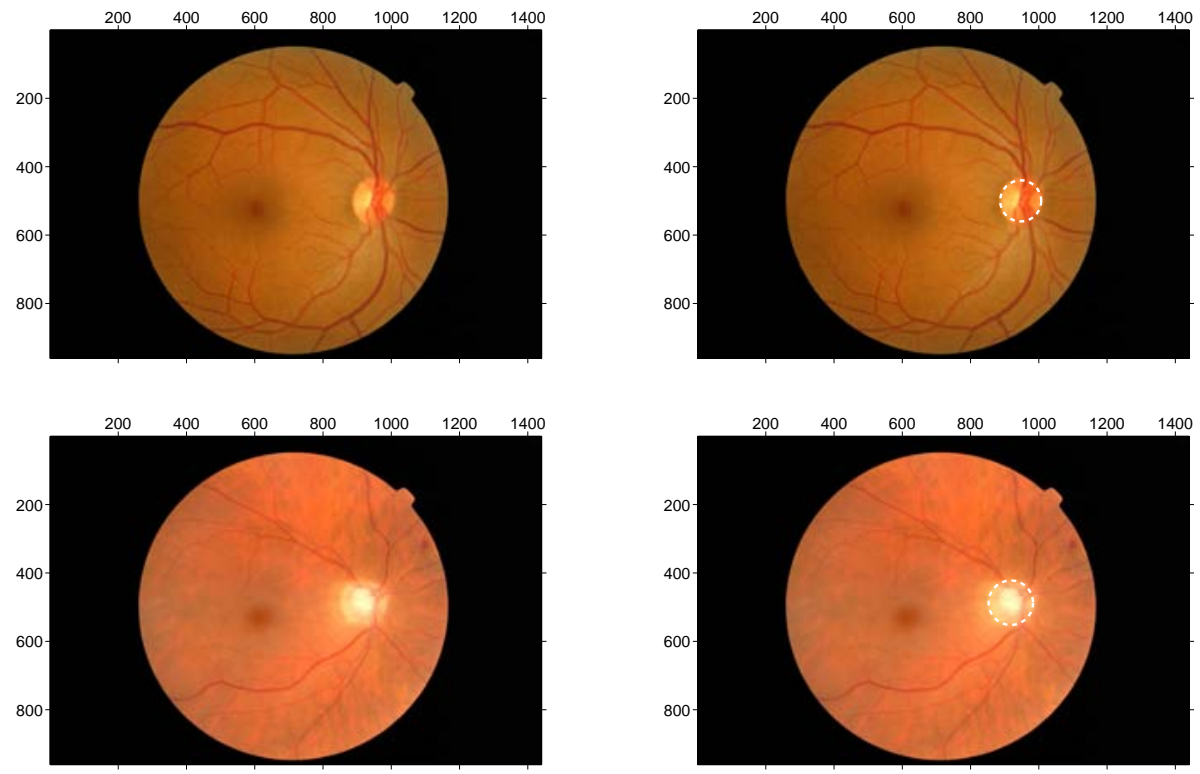

Figure 7: Images of eye fundus. For the detection, the radius range varies from 30 to 80 pixels.

In a slightly more complicated example with the presence of exudates (Fig. 8), the algorithm is still able to detect the optic disk. Its circular shape compensates for a lower value of the gradient. We conclude on this example the ability for the circlet transform to detect at least a single circle.

\subsection{Astronomy}

Counting craters is a method for estimating the age of a planet's surface (Kerr, 2006). We selected two zones from the Moon image and detected a number of craters in a specified radius range (Fig. 9 and 11). The softthresholding process as proposed here allows to remove circles with the same 

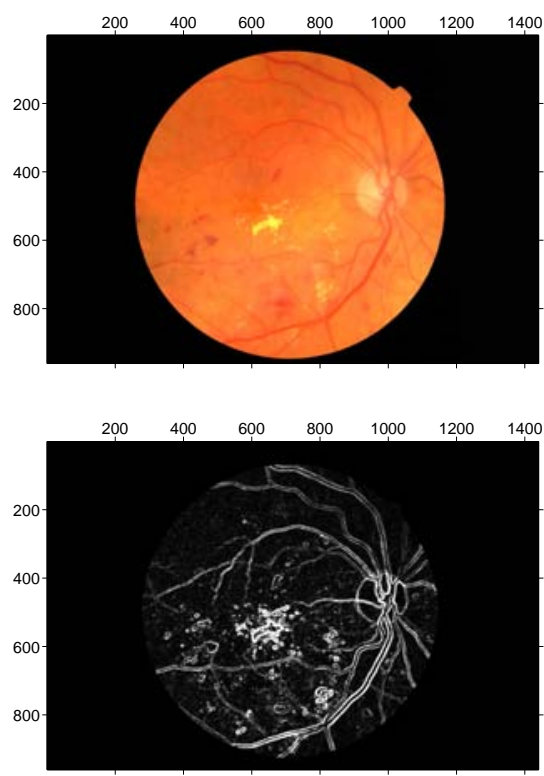
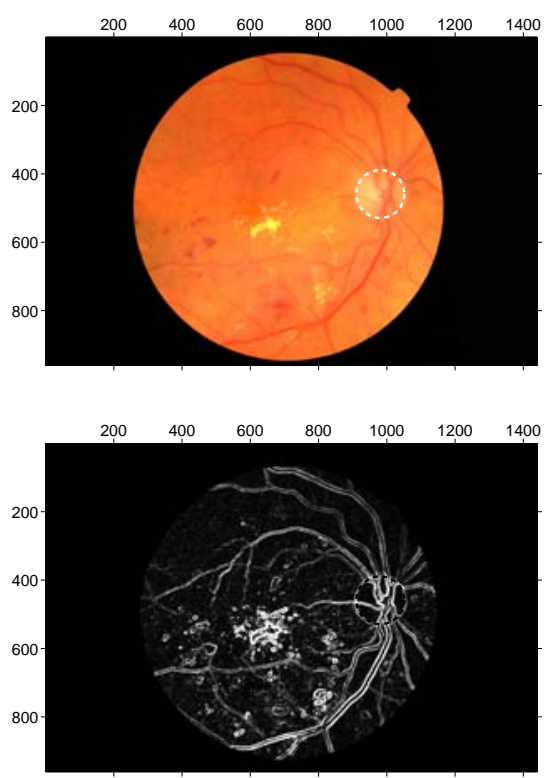

Figure 8: Images of a eye fundus (top) and the associated gradient map (bottom). The blood vessels and exudates (see Fig. 1) have large gradients. For the detection, the radius range varies from 30 to 80 pixels.

radii and slightly different positions. Within the specified range, the craters are indeed well detected (Fig. 9, right). However, some spurious events are also selected (Fig. 10). These are due to the combination of the shallow sun and the topography. We indicate in the discussion part how the artifacts could be removed.

The application on a second moon image leads to similar conclusions (Fig 11). On the crater on the bottom left side, the shape is rather elliptic. In that case, two significant circlets were detected.

\subsection{Oceanography}

In coastal oceanography, the detection of eddies with a sub-mesoscale structure (20-100 km) is a key element for a better understanding of the surface circulation. On remote sensing images, tracers such as the Sea-Surface Temperature (SST) or the chlorophyll maps, are used to reveal the ocean circulation. The underlying assumption is a high correlation between the tracer and the velocity field (Sugimura et al., 1984; Borisov and Monin, 1989; Essen, 1995). The eddies are generally detected either on spatial or temporal gradi- 

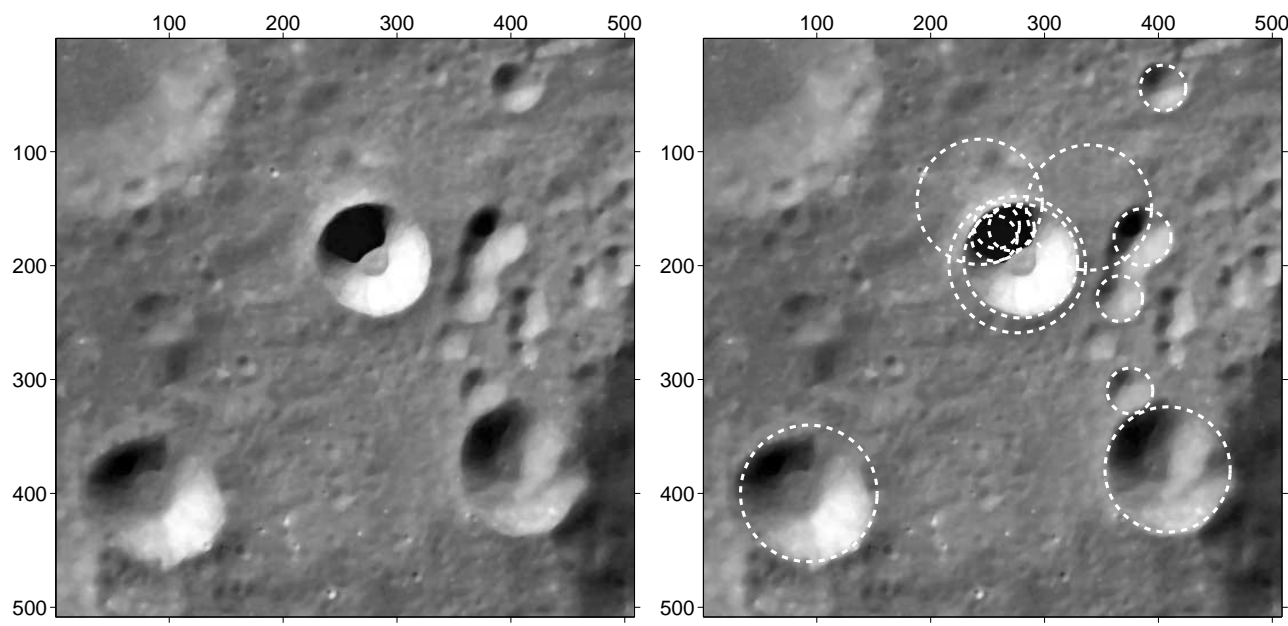

Figure 9: Satellite image from the moon (left), with the first 13 selected circlets (right). The radius range varies from 25 to 60 pixels.
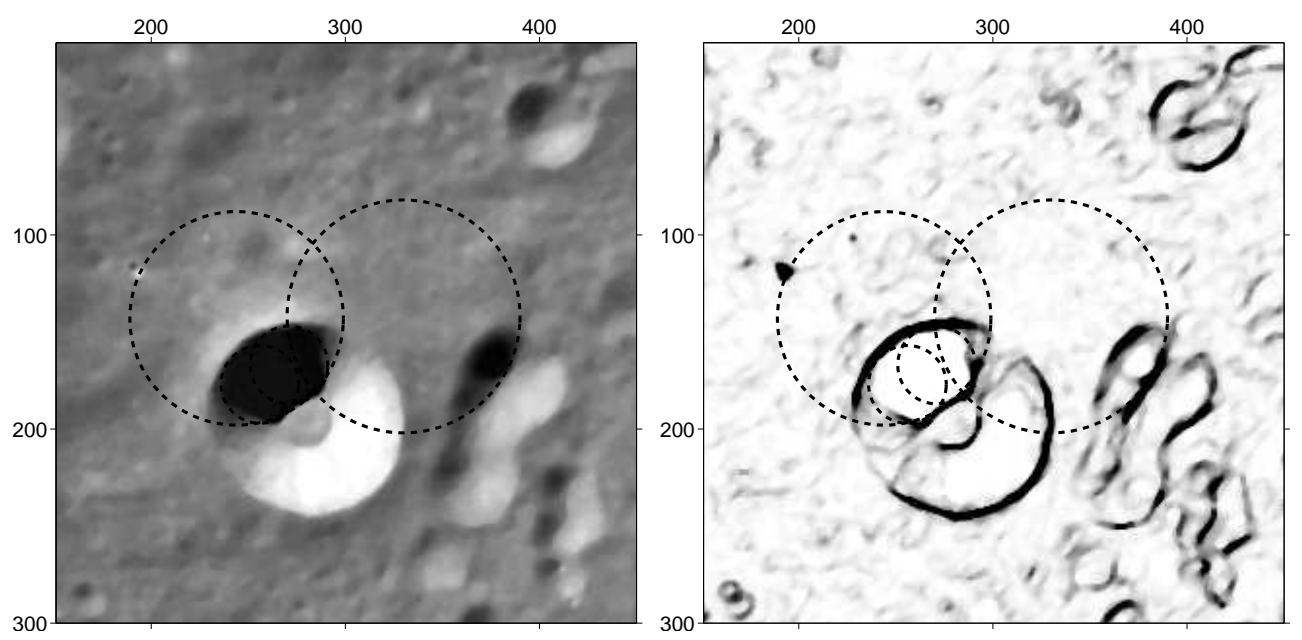

Figure 10: Zoom from the previous figure, where only circlets considered as artifacts are represented, superimposed on the image (left) and its gradient (right). These circlets are tangent to arc lines due to the combination of the sun shadow and the topography.

ent maps (Aleksanin and Aleksanina, 2001; Yang, 2000). We refer to Castellani (2000); Fernandes and Nascimento (2006); Hai et al. (2008); D'Alimonte (2009) for a more complete review. Classically, the Hough transform and several other circle or ellipse fitting algorithms are applied to determine the radius and the central position of eddies on binary edge maps (Peckinpaugh 

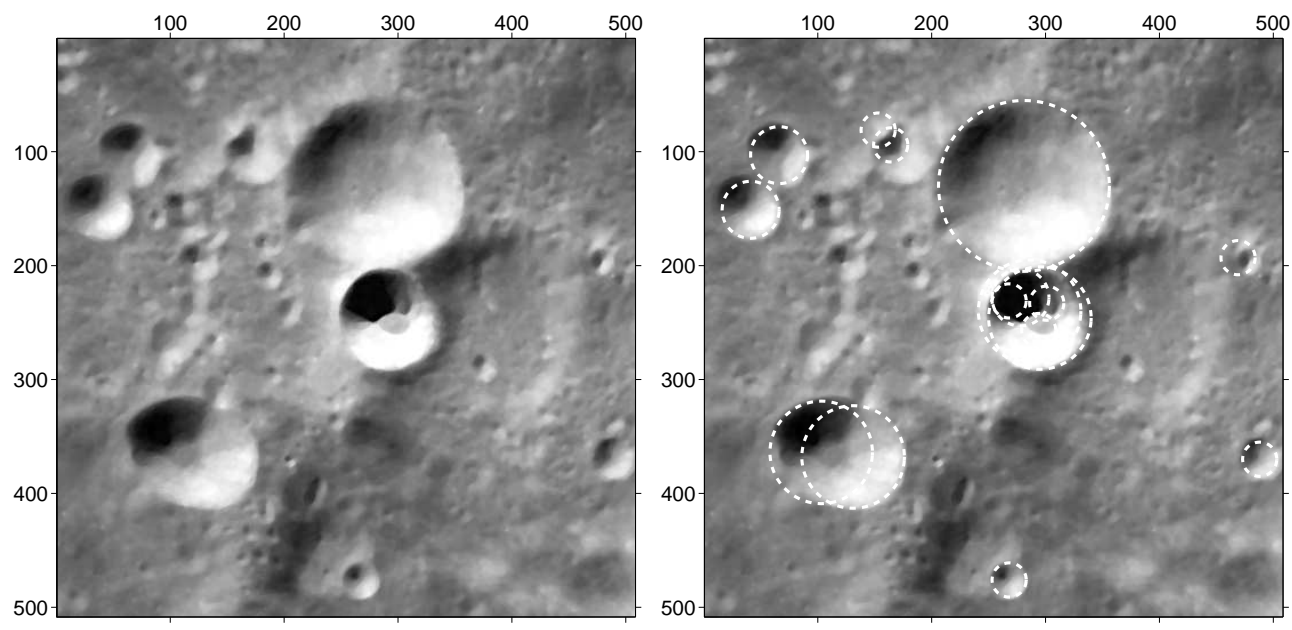

Figure 11: Other satellite image from the moon (left), with the first 16 selected circlets (right). The radius range varies from 15 to 75 pixels.

and Holyer, 1994; Fernandes and Nascimento, 2006).

The main difficulty for detecting eddies is certainly due to the weak and blurry contours. We apply the circlet transform on both synthetic and satellite images.
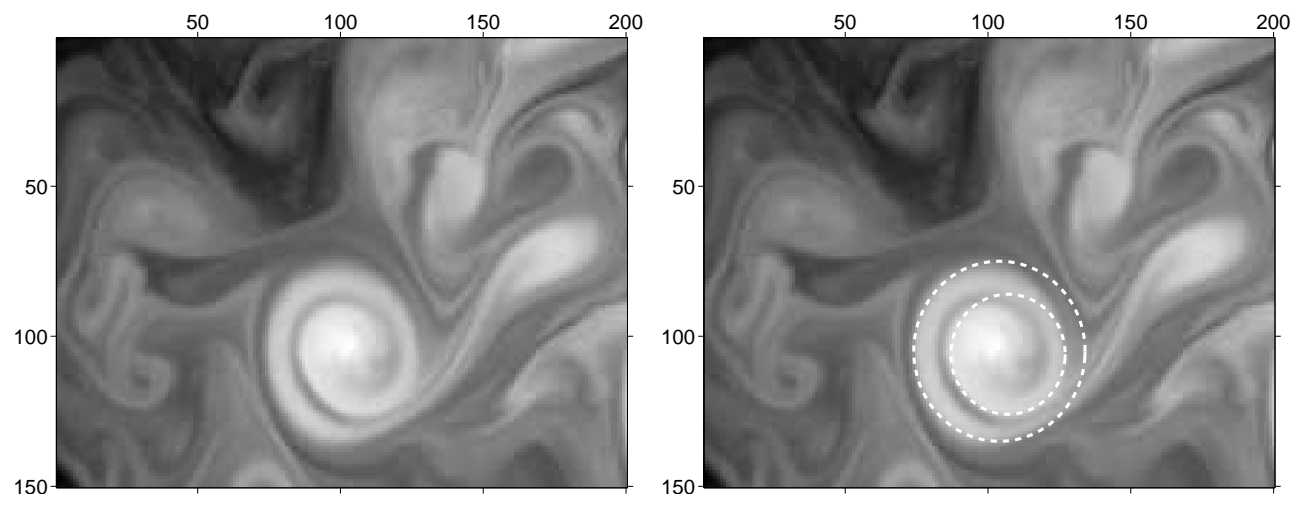

Figure 12: Synthetic SST map. The radius range varies from 10 to 30 pixels.

In the first example, the main circular feature within the radius range is extracted from an SST image computed from a model in a turbulent flow (Fig 12). Two circlets limit the spiral shape. On a larger image, the softthresholding process coupled with the circlet transform is able to detect a 

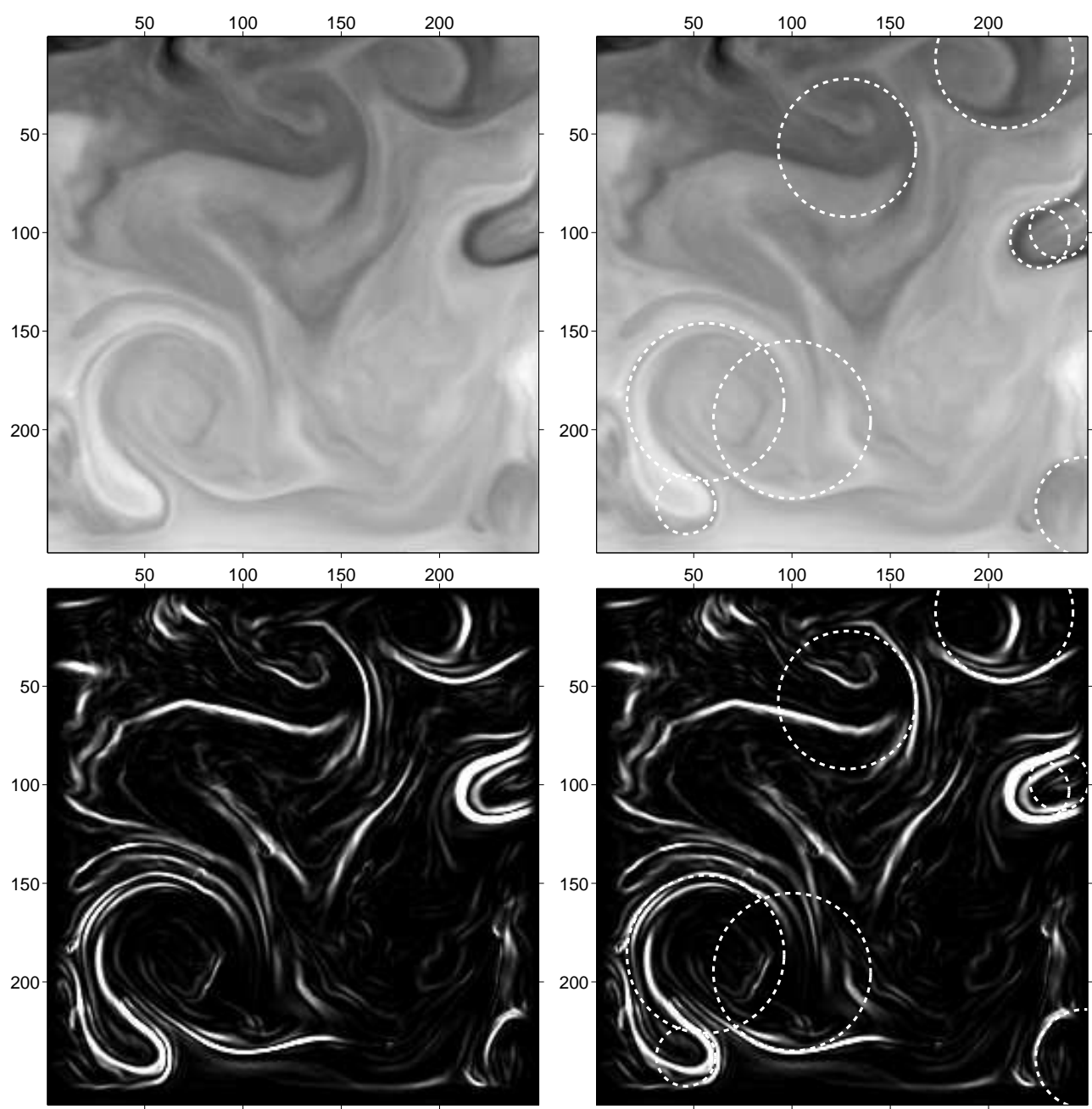

Figure 13: Synthetic SST map (top) and its gradient (bottom). The radius range varies from 15 to 40 pixels.

series of turbulent structures (Fig 13). Further refinements are indicated in the discussion part.

Remote sensing images are usually more blurry (Fig 14, 15 and 16). The circlet transform is able to detect the main eddy on the SST images. For the chlorophyll map, the first four circlet elements are related to spiral features: the chlorophyll filament is trapped in a cyclonic eddy (Fig. 16). The user has to specify the number of selected circlets. A broader selection simply consists of continuing the iterative thresholding process. 

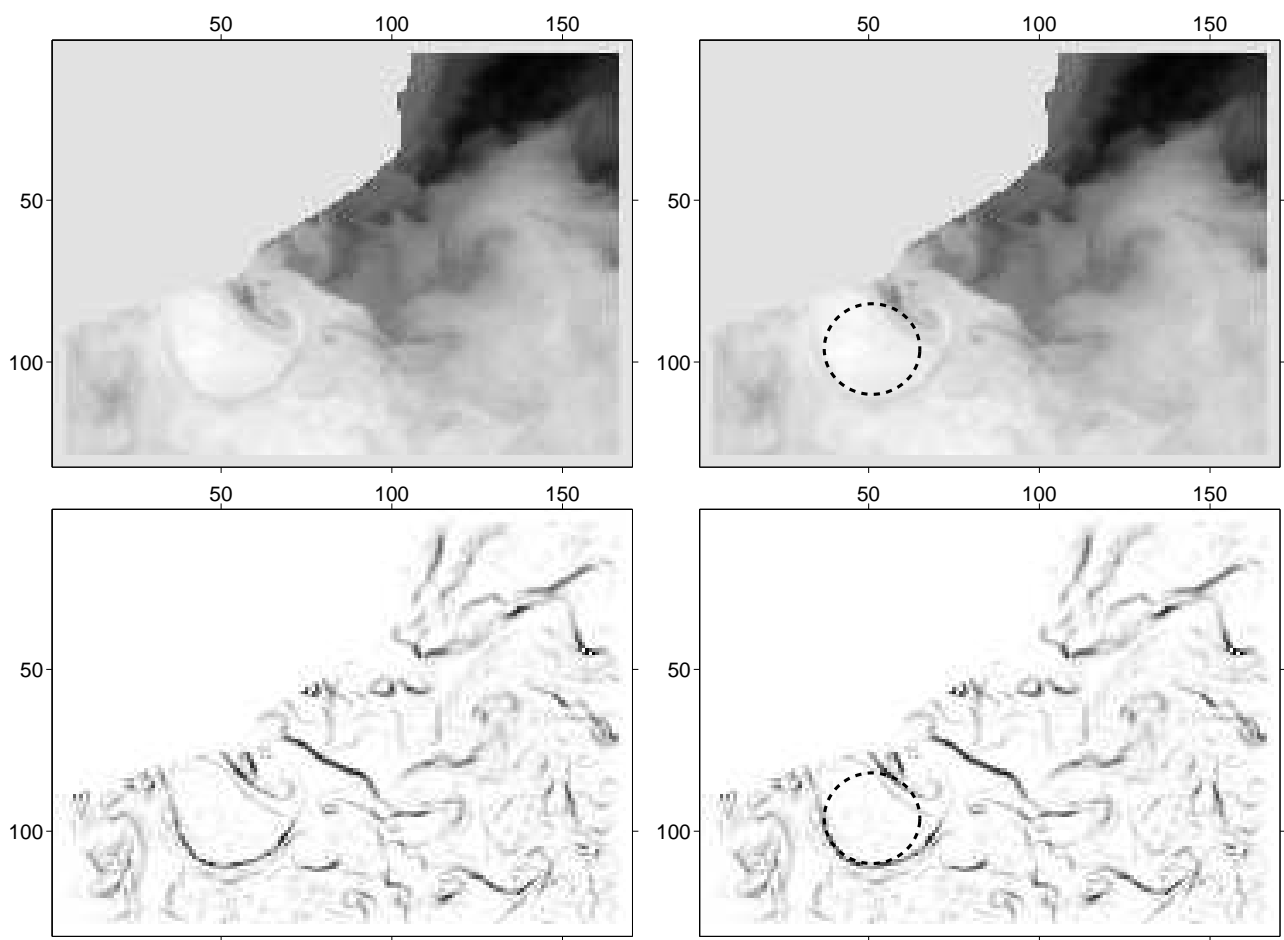

Figure 14: Remote sensing SST image in the Gulf of Lion, North Western Mediterranean Sea (top), and the image gradient (bottom). The radius range varies from 10 to 20 pixels.

\section{Discussion}

Through a series of applications on synthetic and real images, we have shown the capability for the circlet transform to detect circular shapes. The applications are certainly not restricted to the fields presented here. The width of the circlet is important to handle blurry and weak contrasts. The user may easily change the shape of the 1D wavelet under the condition that equation 5 is satisfied. The flexibility also comes from the possibility to specify radius ranges.

The large redundancy of the transform is due to the selection of many radii. With the soft-thresholding approach presented here, this is not really an issue, except for applications on very large images. In practice, these images could be split into smaller images. The size of the overlapping zone should be twice the maximum specified radius to avoid edge effects.

We have observed some spurious detections (e.g. Fig 10). The first possibility would be to measure the local coherency along arcs for the selected 

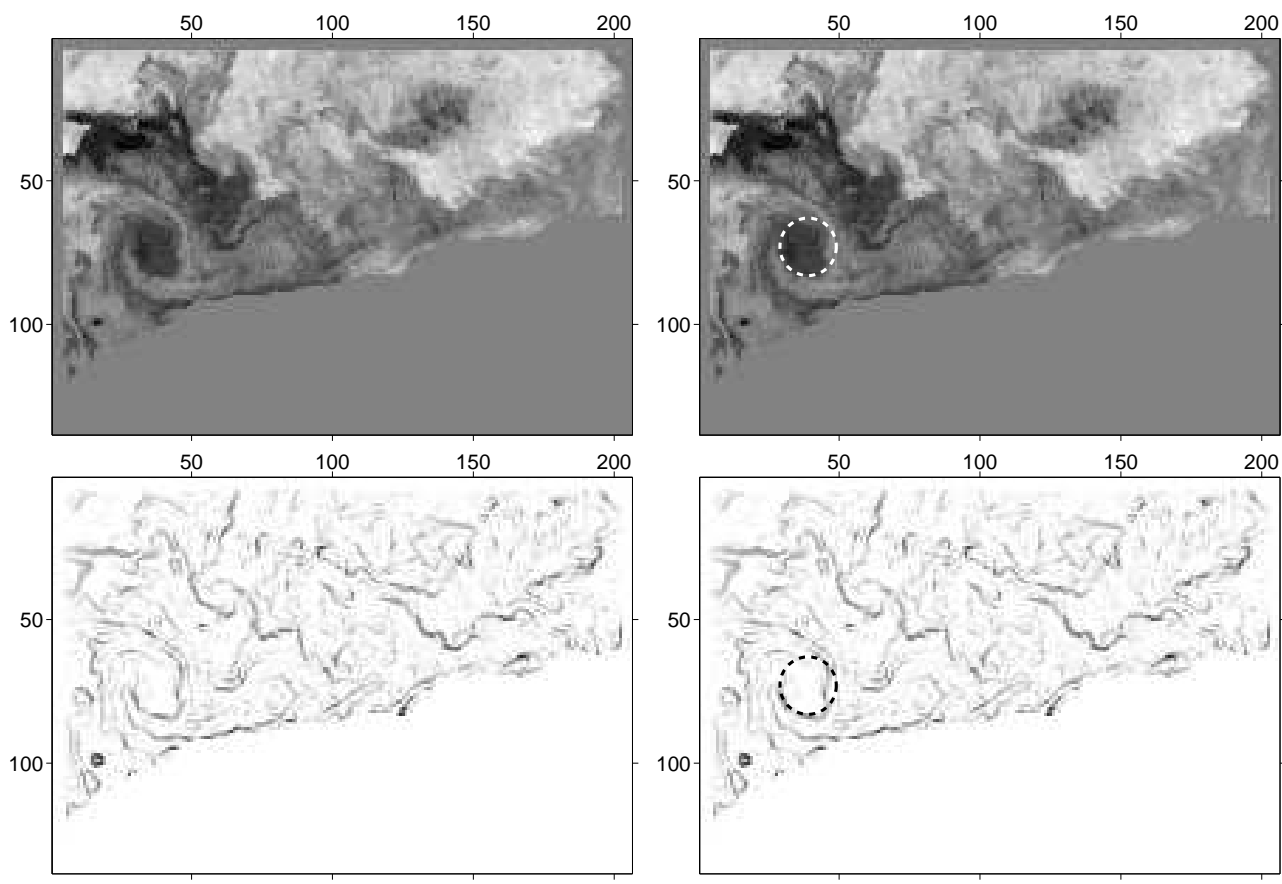

Figure 15: Remove sensing SST image (top) and its gradient (bottom), still in the Gulf of Lion. The radius range varies from 10 to 20 pixels.
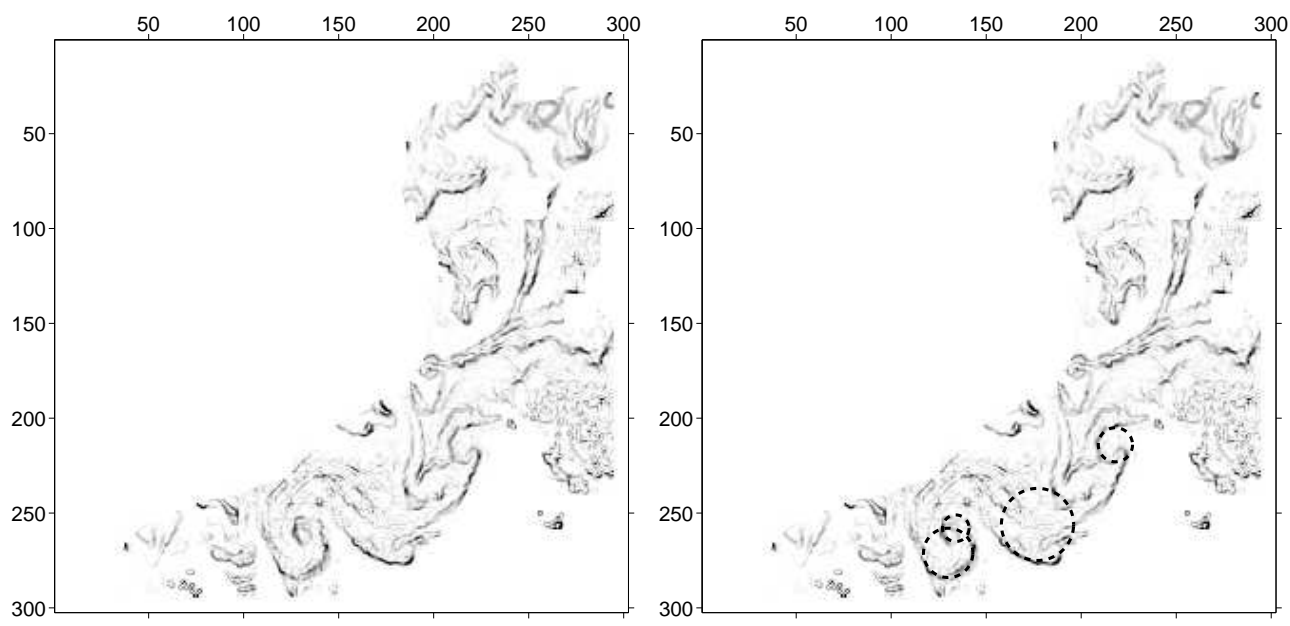

Figure 16: Gradient image of the chlorophyll map (left), with the first 4 more significant circular structures (right). For the selection, the radius range varies from 5 to 20 pixels. The spiral shape on the bottom left is detected by two circlets. 
circlets. This would remove artifacts related to the shadow on the moon image. The other possibility would be to detect circular structures on a sequence of images and track them. Applications in coastal oceanography would be interesting in particular for remote sensing images with weak contrasts (Ma et al., 2006). Other investigations could deal with the multi-scale approach provided by circlets. Currently, we have selected a single scale and various radii. The same analysis could be repeated for different scales. This could deliver a more robust approach if circular shapes are stable at different scales.

\section{Conclusion}

We have presented a flexible method for detecting discontinuities with circular shapes on 2D images. The key property of the transform is certainly the finite frequency aspect of the basis functions. The transform is efficient due to its implementation in the Fourier domain. Combined with a softthresholding algorithm, it appears to successfully detect circular structures on a series of images from different fields. The next step is to track these structures on a sequence of images. This is also potentially a way to remove some spurious detections.

\section{Acknowledgments}

The authors are grateful to the two reviewers for constructive comments. They acknowledge partial funding from the PRECOC project from the FrancoNorwegian Foundation. They thank Jianwei Ma (Tsinghua University, China) for the application on the image from the moon. They also thank Etienne Decencière and Jean-Claude Klein (Mines Paristech) for the medical images. The eye images were kindly provided by the Messidor program partners (see http://messidor.crihan.fr).

\section{A. Appendix - Circular shapes}

We aim at proving that with the definition of Eq. 7, the circlets have circular shapes. In other terms, the functions $G_{k}$ expressed in polar coordinates $(r, \theta)$ should not depend on $\theta$. By definition, we have $(x, y)=(r \cos \theta, r \sin \theta)$. The inverse Fourier transform of $G_{k}$ is 


$$
G_{k}(x, y)=\frac{1}{(2 \pi)^{2}} \iint \mathrm{d} \omega_{1} \mathrm{~d} \omega_{2} e^{-i \omega_{1} x} e^{-i \omega_{2} y} e^{i|\omega| r_{0}} \cdot F_{k}(|\omega|) .
$$

In polar coordinates, $G_{k}$ is expressed as

$$
G_{k}(r, \theta)=\frac{1}{(2 \pi)^{2}} \iint \mathrm{d} \omega_{1} \mathrm{~d} \omega_{2} e^{-i \omega_{1} r \cos \theta} e^{-i \omega_{2} r \sin \theta} e^{i|\omega| r_{0}} \cdot F_{k}(|\omega|) .
$$

We apply a second change of variables from $\left(\omega_{1}, \omega_{2}\right)$ to $(|\omega|, \alpha)$, where $\left(\omega_{1}, \omega_{2}\right)=(|\omega| \cos \alpha,|\omega| \sin \alpha)$, leading to

$$
\begin{aligned}
G_{k}(r, \theta) & =\frac{1}{(2 \pi)^{2}} \iint \mathrm{d}|\omega| \mathrm{d} \alpha|\omega| e^{-i|\omega| r(\cos \theta \cos \alpha+\sin \theta \cos \alpha)} e^{i|\omega| r_{0}} \cdot F_{k}(|\omega|) \\
& =\frac{1}{(2 \pi)^{2}} \iint \mathrm{d}|\omega| \mathrm{d} \alpha|\omega| e^{-i|\omega| r \cos (\theta-\alpha)} e^{i|\omega| r_{0}} \cdot F_{k}(|\omega|) \\
& =\frac{1}{(2 \pi)^{2}} \iint \mathrm{d}|\omega| \mathrm{d} \xi|\omega| e^{-i|\omega| r \cos \xi} e^{i|\omega| r_{0}} \cdot F_{k}(|\omega|)
\end{aligned}
$$

In equation 11, we use the change of variables $\xi=\theta-\alpha$, where $\xi$ is integrated between 0 and $2 \pi$. Eq. 11 does not depend anymore of $\theta$ : in other terms, $G_{k}$ have circular shapes. As all other circlets can be deduced from a shift or by changing the radius or the frequency content of the original circlet, they all have circular shapes.

\section{References}

Aleksanin, A. I., Aleksanina, M. G., 2001. Quantitative analysis of thermal sea surface structures on NOAA IR-images. Proceedings of the International Symposium CREAMS, Vladivostok, 158-165.

Ayala-Ramirez, V., Garia-Capulin, C. H., Perez-Garcia, A., and SanchezYanez, R. E., 2006. Circle detection on images using genetic algorithms. Pattern Recognition Letters 27, 652-657.

Ballard, D. H., 1981. Generalizing the Hough Transform to Detect Arbitrary Shapes Pattern Recognition, 13 (2), 111-122.

Borisov, S. B., Monin, A. S., 1989. About temperature and current correlation in the ocean. Transactions USSR Academy of Science 306, 1230-1233. 
Candes, E., Donoho, D., 2004. New tight frames of curvelets and optimal representations of objects with piecewise $\mathrm{C}^{2}$ singularities: Communication on Pure and Applied Mathematics, 57, 219-266.

Candes, E., Demanet, L., Donoho, D., Ying, L., 2005. Fast discrete curvelet transforms. Multiscale Modeling Simulation 5, 861-899.

Castellani, M., 2005. Automatic detection of Mediterranean water eddies from satellite imagery of the Atlantic ocean, Elsevier Ltd. 163-170.

D'Alimonte, D., 2009. Detection of mesoscale eddy-related structures through iso-SST patterns. Geoscience and Remote Sensing Letters 6, 189193.

Daubechies, I., 1992. Ten lectures on wavelets. SIAM, Philadelphia.

Davies, E. R., 1997. Machine vision: theory, algorithms, practicabilities. Academic Press, San Diego.

Donoho, D., 1995. De-noising by soft-thresholding. IEEE Transactions on information theory, 41 (3), 613-627.

Duda, R. O. and Hart, P. E., 1972. Use of the Hough Transformation to Detect Lines and Curves in Pictures Comm. ACM, 15, 11-15

Essen, H., 1995. Geostrophic surface current as derived from satellite SST images and measured by a land based HF radar. International Journal of Remote Sensing 16, 239-256.

Fernandes, A., Nascimento, S., 2006. Automatic water eddy detection in SST maps using random ellipse fitting and vectorial fields for image segmentation. Lecture Notes in Computer Science 4265, 77-88.

Hai, J.,Xiaomei, Y., Jianming G., Zhenyu, G., 2008. Automatic eddy extraction from SST imagery using artificial neural network. The International Archives of the Photogrammetry, Remote Sensing and Spatial Information Sciences XXXVII. Part B6b, 279-282.

Iivarinen, J., Peura, M., Sarela, J., and Visa, A., 1997. Comparison of combined shape descriptors for irregular objects. In: Proc. 8th British Machine Vision Conf., Cochester, U.K., 430-439. 
Illingworth, J., and Kitttler, J., 1988. A survey of the Hough transform. Comput. Vision Graphics Image Process., 44, 87-116.

Jeong, J., Hussain, F., 1995. On the identification of a vortex. Journal of Fluid Mechanics 285, 69-94.

Kerr, R. A., 2006. Who can read the Martian clock? Science, 206 (5777), 1132-1133.

Kiryati, N., and Bruckstein, A. M., 1991. Antialiasing the Hough transform CVGIP: Graphical models and image processing, 53 (3), 213-222.

Lam, W. C. Y., and Yuen, S. Y., 1996. Efficient technique for circle detection using hypothesis filtering and Hough transform. IEE Proc.-Vis. Image Signal Process., 143 (5), 292-300.

Leavers, V., 1993. Which Hough transform? Comput. Vision Graphics Image Process., 58, 250-264.

Ma, J., Antoniadis, A., Le Dimet, F.-X., 2006. Curvelet-based snake for multiscale detection and tracking of geophysical fluids. IEEE Transactions on Geoscience and Remote Sensing 44, 3626-3638.

Ma, J. and Plonka, G., 2010. The curvelet transform: a review of recent applications: To appear in IEEE Signal Processing Magazine, 27.

Maître, H., 1985. A review on Hough transform. Traitement Signal 2 (4), 305-317.

Mallat, S., 1996 Wavelets for a vision. Proceedings on the IEEE, 84 (4), 604-614.

Montgomery, D., 1993. Clinical disk biometry in early glaucoma. Ophthalomoly, 100, 52-56.

Peckinpaugh, S. H., Holyer, R. J., 1994. Circle detection for extracting eddy size and position from satellite imagery of the ocean. IEEE Transactions on Geoscience and Remote Sensing 32, 267-273.

Song, J., and Lyu, M. R., 2005. A Hough transform based line recognition method utilizing moth parameter space and image space. Pattern Recognition, 38, 539-552. 
Sugimura, T., Tanaka, S., Hatakeyama, Y., 1984. Surface temperature and current vectors in the sea of Japan from NOAA-7 AAVHRR data. Remote sensing shelf sea hydrodynamics, Proceedings 15 International Liege. Colloquium, 133-147.

Wackernagel, H., 2003. Multivariate Geostatistics: an introduction with applications. Springer-Verlag, Berlin, 3rd edition.

Yang, Q., Parvin, B., Mariano, A., 2000. Singular features in Sea Surface Temperature data. 15th International Conference on Pattern Recognition $1,516-520$. 